\title{
Efetividade da Compensação Ambiental Monetária no Brasil
}

\author{
Alexandre Nascimento de Almeida ${ }^{1}$, Emily Mendes Xavier ${ }^{1}$, \\ Antônio Felipe Couto Junior ${ }^{1}$, Ludgero Cardoso Galli Vieira ${ }^{1}$ \\ ${ }^{1}$ Universidade de Brasília - UnB, Brasília/DF, Brasil
}

\begin{abstract}
RESUMO
No Brasil, a compensação ambiental monetária ocorre em situações em que um impacto ambiental de um projeto não pode ser evitado e nem mitigado, restando ao empreendedor a obrigatoriedade de apoiar a implantação e manutenção de Unidade de Conservação (UC). Os critérios para a escolha da área a ser beneficiada estão relacionados à sua conexão espacial ou funcional com a área impactada. O objetivo deste estudo foi avaliar a efetividade da compensação ambiental monetária, avaliando a conexão espacial e funcional na aplicação dos recursos desse instrumento. Para tanto, compararam-se os recursos gerados da compensação ambiental a serem aplicados no município de ocorrência do empreendimento, bem como verificou-se se as áreas afetadas e beneficiadas pertenciam ao mesmo bioma. Os resultados indicaram que a compensação ambiental monetária não costuma ocorrer no mesmo município do empreendimento. Porém, em 96\% dos casos, ela tende a ocorrer no mesmo bioma.
\end{abstract}

Palavras-chave: avaliação de impacto ambiental, compensação ambiental monetária, gestão ambiental, Unidade de Conservação.

\section{Effectiveness of Monetary Environmental Compensation in Brazil}

\begin{abstract}
In Brazil, monetary environmental compensation occurs in situations in which a project environmental impact cannot be avoided or mitigated, resting to the entrepreneur an obligation to support the implementation and maintenance of a Protected Area. The criteria for choosing the area to be benefited are related to spatial and functional connection with the same. This study aimed to evaluate the effectiveness of monetary environmental compensation, evaluating the spatial and functional connection in the application of the instrument resources. Under these circumstances we assessed if resources generated from environmental compensation tend to be applied in the project site, as well as, if the affected areas benefited belong to the same biome. Results indicated that monetary environmental compensation does not usually occur in the project nearby area; however, in $96 \%$ of cases it tends to occur in the same biome.
\end{abstract}

Keywords: environmental impact assessment, monetary environmental compensation, environmental management, Conservation Unit.

\section{INTRODUÇÃO}

A gestão ambiental pode ser entendida como o conjunto de procedimentos e atividades que objetiva conciliar desenvolvimento com qualidade ambiental.
Ela tem como papéis punir quem polui e preservar os recursos naturais, monitorando e otimizando a sua utilização, o que resulta na redução dos danos ou 
problemas causados pelas ações antrópicas (Sánchez, 2008).

Até a década de 1970, as ações de gestão ambiental possuíam um caráter prioritariamente corretivo e com iniciativas fragmentadas. A partir da Conferência Mundial sobre o Homem e Meio Ambiente, realizada em 1972, vários países começaram a adotar um enfoque preventivo e integrado em sua política ambiental (Barbieri, 2007).

O início de ações ambientais preventivas no Brasil decorreu de iniciativas de zoneamento industrial, em uma perspectiva de separação entre uso do solo industrial e áreas residenciais em 1978 (Sánchez, 2008). A consolidação dessa abordagem no país ocorreu com a Política Nacional do Meio Ambiente, por meio da exigência do Estudo de Impacto Ambiental - EIA para o licenciamento de todas as atividades capazes de causar impactos ambientais significativos (Brasil, 1981).

O EIA é um instrumento para prever e avaliar os impactos negativos de um projeto sobre o meio ambiente e identificar alternativas para evitá-los antes da implementação do projeto (Barbieri, 2007). Porém alguns impactos ambientais não podem ser evitados e outros, mesmo que mitigados, podem ainda ter magnitude muito elevada. Nessas situações, a legislação prevê a adoção de medidas compensatórias (Brasil, 2000).

Geralmente, dois tipos de compensação ambiental são distinguidos em diversos países: 1) pela adoção de ações diretas no meio ambiente; ou 2) pelo pagamento monetário que pode ser investido no meio ambiente. A primeira consiste em proporcionar uma restauração ambiental ou implementar outras ações que proporcionem benefícios de restauração. A compensação monetária consiste em pagamento em dinheiro em decorrência dos danos causados (Gastineau \& Taugourdeau, 2014).

Na legislação brasileira a compensação ambiental monetária está prevista no artigo 36 da lei 9.985, de 2000, que instituiu o Sistema Nacional de Unidades de Conservação da Natureza (SNUC). Nos casos de licenciamento ambiental de empreendimentos de impacto ambiental significativo, assim considerado pelo órgão ambiental competente, com fundamento em Estudo de Impacto Ambiental e respectivo relatório - EIA/RIMA, o empreendedor é obrigado a apoiar a implantação e manutenção de Unidade de Conservação (UC) do Grupo de Proteção Integral, de acordo com o disposto nesse artigo e na regulamentação dessa lei (Brasil, 2000).

Segundo Faria (2008), a compensação ambiental monetária é definida como mecanismo financeiro com a finalidade de compensar os impactos ambientais negativos não mitigáveis ocorridos ou previstos no EIA para obtenção de licença ambiental. Para Ten Kate et al. (2004), a compensação ambiental monetária é considerada o último recurso para contrabalancear os impactos ambientais negativos causados pelos empreendimentos como, por exemplo, perda de biodiversidade e perda de áreas representativas dos patrimônios histórico e arqueológico, de modo a garantir que não haja perda líquida de biodiversidade.

Desde a década de 1970, em muitos países tem crescido a importância da compensação ambiental na decisão de conceder a licença ambiental (Rundcrantz, 2006). Porém, como bem colocado por Cowell (1997), continua a ser discutido em que medida a perda dos valores ambientais pode, eventualmente, ser restaurada ou revertida pela compensação monetária. Para Villarroya \& Puig (2010), a compensação ambiental deve compensar o impacto ambiental da atividade humana, evitando uma perda líquida na base de recursos ambientais, e não indenizar por seu esgotamento. Na visão de Xie et al. (2013), a compensação ambiental é um tema interdisciplinar no qual, durante as últimas décadas, nenhum consenso foi alcançado em termos de investigação.

Conforme Sánchez (2008), os princípios que norteiam a compensação ambiental monetária devem ser: 1) proporcionalidade entre o dano causado e a compensação exigida; 2) conexão espacial: preferência por medidas que possam ser implementadas em área contígua à área afetada; 3) conexão funcional: preferência por medidas compensatórias que representem a reposição ou a substituição das funções ou dos componentes ambientais afetados.

Objeto de muitas disputas e recente modificação se refere ao princípio da proporcionalidade, pois era prevista compensação mínima referente a $0,5 \%$ do valor do empreendimento (Brasil, 2006), o que foi considerado inconstitucional pelo Supremo Tribunal Federal, por não haver uma correspondência com o impacto previsto pelo projeto.

Enquanto o atendimento do princípio da proporcionalidade tem sido amplamente discutido na literatura (Domingues \& Carneiro, 2010; Bechara, 
2007; Faria, 2008), pouco é se fala sobre a adequação aos princípios da conexão espacial e funcional, embora a Resolução Conama n. 371, no seu artigo $9^{\circ}$, deixe claro o intuito de compensar áreas que possuam uma conexão funcional e, principalmente, uma conexão espacial com os impactos gerados.

Conforme a resolução Conama supracitada, existindo uma ou mais UC ou zonas de amortecimento afetadas diretamente pelo empreendimento ou atividade a ser licenciada, independentemente do grupo a que pertençam, deverão elas serem beneficiárias de recursos da compensação ambiental, considerando-se, entre outros, os critérios de proximidade, dimensão, vulnerabilidade e infraestrutura existentes (Brasil, 2006).

Porém, devido à possibilidade de não existir UC ou zonas de amortecimento afetadas pelo empreendimento, inviabilizando-se o atendimento do princípio da conexão espacial, a legislação passa a priorizar áreas que possuam as mesmas funções ou os mesmos componentes ambientais afetados, obedecendo ao princípio da conexão funcional. Inexistindo UC ou zona de amortecimento semelhante à afetada, parte dos recursos oriundos da compensação ambiental deverá ser destinada à criação, implantação ou manutenção de UC do Grupo de Proteção Integral localizada preferencialmente no mesmo bioma e na mesma bacia hidrográfica do empreendimento ou atividade licenciada (Brasil, 2006).

Por fim, a Resolução Conama n. 371 de 2006 (Brasil, 2006), no seu artigo $9^{\circ}$, em parágrafo único, possibilita a destinação dos recursos da compensação ambiental para UC do grupo de proteção integral em qualquer região do território brasileiro, portanto não obedecendo aos princípios da conexão funcional e espacial, permitindo grande flexibilidade na aplicação dos recursos pelo órgão ambiental

A flexibilização da legislação brasileira na aplicação dos recursos da compensação e o fato de o empreendedor não ser obrigado a se envolver diretamente nas medidas de compensação podem formar uma cultura em que são aceitos pagamentos financeiros como solução de obrigações ambientais e incentivar um descompromisso com os princípios que norteiam a compensação ambiental (Bezerra, 2007), gerando muitas críticas a essa política.

Assim, o objetivo deste trabalho foi avaliar o cumprimento dos princípios de conexão espacial e funcional pela compensação ambiental monetária no apoio de implantação e manutenção de UC, conforme o artigo 36 da lei n. 9.985, de 2000, a qual instituiu o SNUC (Brasil, 2000).

\section{MATERIAL E MÉTODOS}

\subsection{Dados utilizados}

Os dados desta pesquisa foram coletados junto a Caixa Econômica Federal e se referem ao fluxo dos recursos financeiros gerados para compensação ambiental a partir de uma amostra de 110 empreendimentos que tiveram a aplicação dos recursos financeiros da compensação na modalidade indireta. Nessa modalidade, o empreendedor deve efetuar o depósito dos recursos da compensação em contas escriturais na instituição bancária contratada pelo Instituto Chico Mendes (ICMBIO), no caso, a Caixa Econômica Federal.

A partir dos dados referentes à aplicação de recursos entre o período de 2009 e 2013 e de informações obtidas junto ao Instituto Brasileiro de Meio Ambiente e dos Recursos Naturais Renováveis - IBAMA (2014), Instituto Chico Mendes - ICMBIO (2014) e Instituto Brasileiro de Geografia e Estatística IBGE (2014), foram identificados o município e o montante de recurso gerado por cada empreendimento, bem como, o município e o bioma da unidade de conservação UC de destino do recurso da compensação ambiental. Isso possibilitou caracterizar o fluxo dos recursos da compensação ambiental. A Figura 1 tipificou os empreendimentos analisados e ilustrou a sua distribuição espacial conforme os municípios e biomas brasileiros.

\subsection{Instrumental analítico}

A avaliação da conexão espacial considerou a proximidade entre o impacto gerado e a destinação dos recursos financeiros da compensação ambiental. Para tanto, a premissa adotada foi a de que recursos investidos, mesmo que apenas parte deles, no mesmo município ao do impacto gerado devem atender ao princípio da conexão espacial.

Já para a conexão funcional, a premissa adotada foi a de que o investimento dos recursos da compensação ambiental no mesmo bioma afetado pelo empreendimento deve atender a esse princípio. Para essa análise, os 


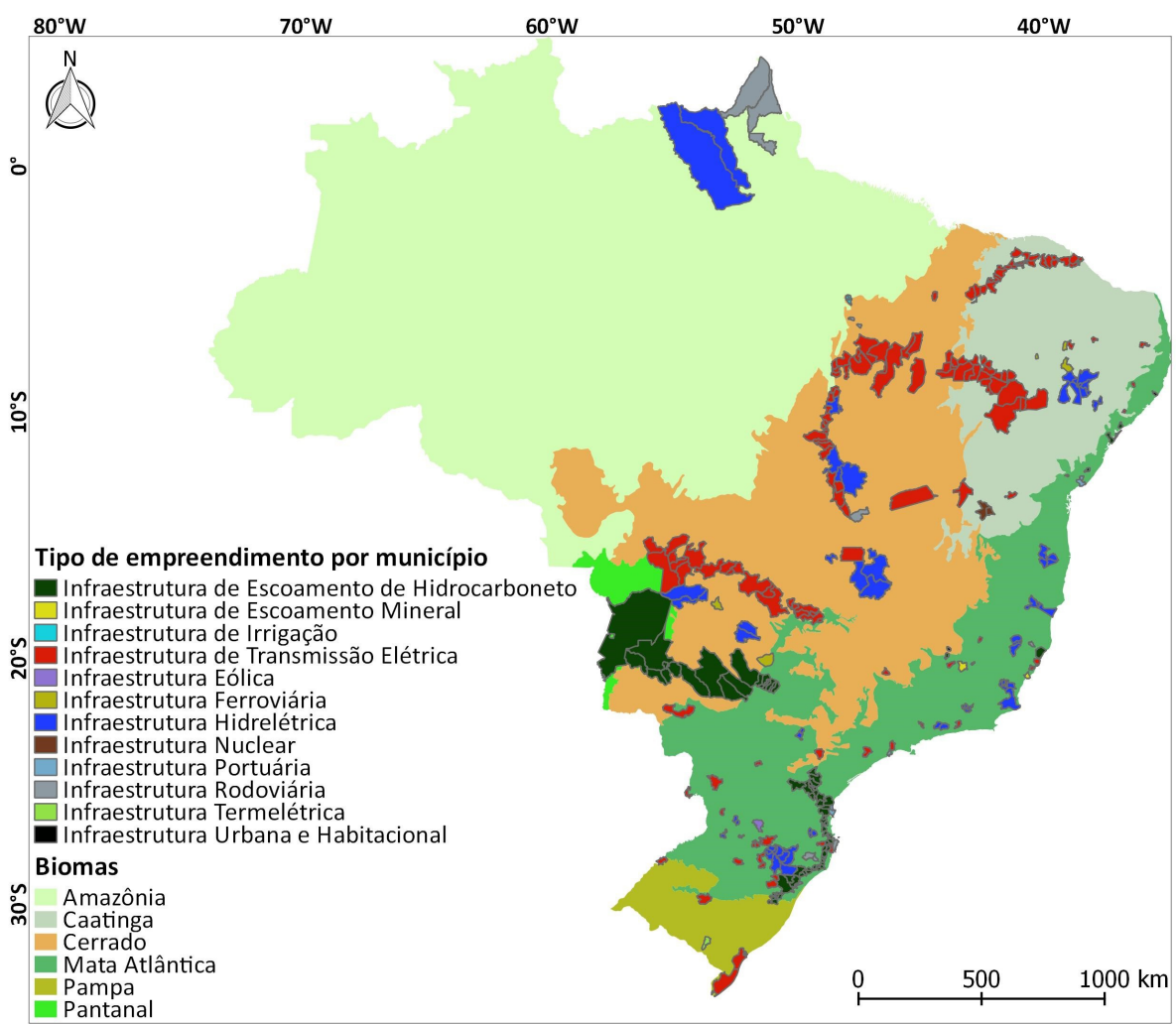

Figura 1. Tipificação e distribuição espacial dos empreendimentos analisados. Fonte: Dados utilizados na pesquisa. Figure 1. Classification and spatial distribution of the projects analyzed. Source: Data used in the research.

biomas considerados foram: Mata Atlântica, Amazônia, Cerrado, Caatinga, Pampa e Pantanal.

Devido aos dados utilizados serem em uma escala nominal, permitindo apenas o cálculo da frequência deles, a análise estatística empregada considerou o teste não paramétrico da Binomial.

Conforme Pestana \& Gageiro (2005), o teste da Binomial se aplica a uma amostra independente em que a variável qualitativa é dicotômica, aquela cujas características são designadas por sucesso e insucesso.

Portanto, a avaliação da conexão espacial obedeceu às seguintes hipóteses:

$\mathrm{H}_{0}$ : a maioria dos empreendimentos tem por destinação a compensação ambiental para UC no mesmo município ou em município limítrofe (condição de sucesso do teste);

$\mathrm{H}_{1}$ : a maioria dos empreendimentos não tem destinação da compensação ambiental para UC no mesmo município ou em município limítrofe (condição de insucesso do teste).
Para a avaliação da conexão funcional as hipóteses testadas foram:

$\mathrm{H}_{0}$ : a maioria dos projetos consegue destinar seus recursos para regiões do mesmo bioma (condição de sucesso do teste);

$\mathrm{H}_{1}$ : a maioria dos projetos não consegue destinar seus recursos para regiões do mesmo bioma (condição de insucesso do teste).

O teste da Binomial comparou as frequências observadas com as que se espera obter numa distribuição binomial e identificou se existe diferença estatística entre elas ao nível de significância de até 5\% (Pestana \& Gageiro, 2005). O software utilizado para aplicação do teste foi o SPSS versão 20.

\section{RESULTADOS E DISCUSSÕES}

Dos 110 empreendimentos avaliados, em 68\% dos casos a compensação ambiental foi direcionada para um município diferente do local do empreendimento. 
O teste da Binomial rejeitou $\mathrm{H}_{0}(p=0,01)$ para a avaliação da conexão espacial, indicando que a compensação ambiental tende a ocorrer em município diferente do local onde ocorre o impacto ambiental (Figura 2).

Para a conexão funcional, apenas em $4 \%$ dos casos a aplicação do recurso da compensação ambiental não ocorreu no mesmo bioma. Para essa hipótese, o teste da Binomial não rejeitou $\mathrm{H}_{0}(p=0,01)$. Portanto, embora a compensação ambiental não costume ocorrer no mesmo local do impacto, na grande maioria dos casos ela tende a ocorrer no mesmo bioma e, em geral, contribuindo para preservar os mesmos ecossistemas afetados pelo empreendimento (Figura 2).

Tendo em vista que a legislação ambiental brasileira prioriza a compensação ambiental para implementação e manutenção de UC, não surpreendeu a dificuldade no atendimento do princípio da conexão espacial, pois não são todos os municípios que possuem UC.

Conforme Medeiros \& Young (2011), as UC criadas e administradas pelo governo federal e estadual somaram 698 unidades, enquanto as Reservas Particulares do Patrimônio Natural - RPPN, outras 973, em 2010. Ainda conforme os autores, as informações para as UC municipais ainda são muito dispersas e estimativas conservadoras do Ministério do Meio Ambiente - MMA indicaram existir pelo menos 600 UC municipais. Porém, mesmo considerando todo o somatório de UC, dado a existência de 5.564 municípios no Brasil, pode-se inferir que a abrangência das UC ainda é carente para a cobertura da maioria dos municípios brasileiros.

Em alguns casos, a grande magnitude dos impactos inviabiliza a aplicação dos recursos financeiros da compensação ambiental no local e, por isso, tal instrumento não traz benefícios à biodiversidade (Suvantola, 2005). Porém o empreendedor deverá apresentar em seu projeto medidas para evitar, minimizar e mitigar os danos ambientais. Dunford et al. (2004) corroboraram as dificuldades do alcance da conexão espacial na compensação ambiental, destacando a possibilidade de inviabilidade ecológica ou econômica na restauração de um hábitat do mesmo tipo e qualidade do que foi danificado.

No entanto, implementações de grandes empreendimentos impactam a biodiversidade e a sociedade, que sente os impactos diretos do projeto, tais como ruídos e perda de espaços verdes, e é também privada de serviços ecológicos que melhoram a qualidade de vida. Segundo relatório do IUCN (2012), as comunidades não se sentem satisfeitas com a aplicação dos recursos da compensação ambiental caso esse seja investido em um local a distância superior a $200 \mathrm{~km}$, especialmente devido à não percepção dos serviços ecossistêmicos.

$\mathrm{O}$ vínculo legal da destinação dos recursos da compensação para UC pode incentivar a criação delas pelos municípios e melhorar a qualidade ambiental local, porém a legislação não prevê a possibilidade de aplicação dos recursos em problemas ambientais no local onde ocorre o impacto como, por exemplo, em problemas ambientais ocasionados pela precariedade do saneamento urbano de muitas cidades. Nesse aspecto, deve ser ressaltado que o conceito de ambiente no campo planejamento e gestão ambiental é amplo e inclui tanto a natureza como a sociedade (Sánchez, 2008).

Conforme Leoneti et al. (2011), até 2006, apenas $15 \%$ do esgoto sanitário gerado nas regiões urbanas dos municípios do Brasil era tratado, trazendo muitos problemas de ordem ambiental e social. A compensação ambiental decorrente da perda de um bem natural por meio de melhorias urbanas locais, sob uma óptica mais ampla, dificilmente seria aceito pela sociedade como um todo. Porém, para o público diretamente afetado poderia ser
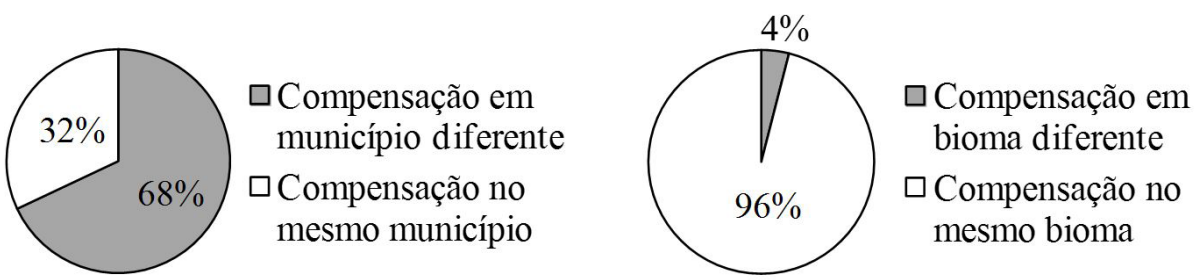

Figura 2. Destino dos recursos da compensação ambiental.

Figure 2. Resource allocation of environmental compensation. 
uma solução plausível, podendo complementar o processo de compensação ambiental.

Ademais, a conexão espacial é fundamental para que haja uma distribuição social dos impactos positivos e negativos do projeto, como uma forma de licença social para a instalação do empreendimento. Caso contrário corre-se o risco de atrasar o licenciamento, podendo levar a intermináveis disputas judiciais do processo. A expressão "licença social" é usada com frequência para designar a aceitação pública de um projeto, independentemente da existência de autorizações ou licenças governamentais (Sánchez, 2008).

A importância dessa licença social é tamanha que Ter Mors et al. (2012) se referiram às medidas de compensação como uma estratégia para reduzir o desequilíbrio entre custos e benefícios locais. Corroborando Ter Mors et al. (2012), Zaal et al. (2014) destacaram a importância da compensação monetária para ajudar a prevenir ou reduzir a oposição do público local à instalação de projetos que enfrentam oposição como, por exemplo, penitenciárias, hidroelétricas, aterros sanitários, entre outros.

Cole (2013) e Gastineau \& Taugourdeau (2014) destacaram a importância da conexão espacial na compensação ambiental monetária, propondo que o valor da compensação não seja determinado apenas em função do custo de alcançar uma equivalência ambiental do ambiente perdido, devendo ser priorizada uma análise dos custos e benefícios da sociedade afetada pelo projeto e não da sociedade como um todo.

Corroborando a importância da conexão espacial, Quétier et al. (2014), Darbi et al. (2009), Mckenney \& Kiesecker (2010) defendem que o objetivo da compensação é, de preferência, atingir ganho de biodiversidade em relação a composição de espécies, estrutura do hábitat, valores e funções dos ecossistemas e uso das pessoas associados à biodiversidade impactada. Ou seja, quando a conexão espacial é alcançada, em geral a conexão funcional também o é, pois são os locais próximos ao impacto que são mais propensos a ter espécies e hábitats semelhantes, a desempenhar papéis funcionais semelhantes.

Apesar de os resultados deste estudo mostrarem que não há conexão espacial entre os impactos do empreendimento e a sua compensação ambiental, os resultados indicaram uma conexão funcional nos investimentos provenientes da compensação.
Nesse aspecto, pode-se afirmar que a cobertura das UC nos biomas brasileiros atende aos princípios da conexão funcional predeterminados. Segundo Medeiros \& Young (2011), ainda que em número suficiente ou inadequadamente implementada, o fato é que as UC estão presentes em todo o território nacional, contribuindo para a conservação de importante parcela de todos os biomas do Brasil.

Conforme Gurgel et al. (2009), a porcentagem da área dos biomas no Brasil protegidos por UC em 2009 foi: Amazônia - 26,2\%; Cerrado - 7,9\%; Mata Atlântica - 7,8\%; Caatinga - 7,3\%; Pantanal - 4,0\%; Pampa - 3,5\%; Área marinha - 1,5\%.

Embora o Brasil, junto com a maioria dos países do mundo, não tenha conseguido alcançar a meta assumida em 2002 na Convenção sobre Diversidade Biológica, que estabeleceu para o país o percentual de $30 \%$ para Amazônia e 10\% para os demais biomas para o ano de 2010, o esforço brasileiro na criação de UC nos últimos anos é notável (Medeiros \& Young, 2011). Enquanto o Brasil tem aproximadamente 16,7\% de seu território continental protegidos em UC, no mundo apenas $12,8 \%$ dos territórios encontram-se atualmente sob proteção legal (WDPA, 2009).

Apesar da tentativa de proporcionar ganhos equivalentes às perdas causadas pelos impactos ambientais por meio da conexão funcional, cada bioma brasileiro apresenta grande diversidade de tipos de vegetação, caracterizadas por diferenças na composição das espécies e estrutura dos ecossistemas (Ribeiro et al., 2009). Dessa forma, mesmo que as compensações ocorram no mesmo bioma, não é assegurado a equivalência ecológica de composição, de estrutura e de função do meio (SBPC \& ABC, 2012), tornando difícil mensurar se os danos ambientais ocorridos são de fato compensados.

Além disso, deve ser levado em conta a opinião de Darbi et al. (2009). O autor ressaltou a importância da compensação ambiental em áreas que não possuam nenhuma conexão com o sítio afetado. Segundo Darbi et al. (2009), a melhoria ambiental é alcançada pela substituição qualitativa ou quantitativa de recursos menos valiosos por recursos mais valiosos, ou seja, assegurar uma compensação ambiental em ecossistemas considerados mais ameaçados ou de maior prioridade que o ecossistema impactado.

Para tanto, é importante o desenvolvimento de métodos bem definidos de valoração ambiental, de modo a aferir o valor ecológico do ecossistema impactado 
e do ecossistema considerado de maior relevância do ponto de vista ambiental, pois todos os ecossistemas têm suas particularidades e são importantes para a manutenção da vida no planeta. A dificuldade de valorar o meio ambiente na busca de um consenso sobre os valores da compensação ambiental monetária sido um dos maiores limitantes na aplicação do instrumento em todo o mundo (Gastineau \& Taugourdeau, 2014; Martin-Ortega et al., 2011).

A dificuldade de estabelecer parâmetros de compensação por meio de técnicas de valoração ambiental foi demonstrada por Martin-Ortega et al. (2011). Os autores mostraram que a compensação monetária decorrente de um vazamento tóxico de uma barragem com rejeitos de uma mina de pirita no Parque Nacional Doñana na Espanha em 1998 pode ter sido insuficiente para compensar as perdas ambientais da sociedade.

Apesar de todos os problemas jurídicos para implementação da compensação ambiental monetária no Brasil, principalmente no alcance do princípio da proporcionalidade entre impacto causado e valor a ser compensado, bem como na dificuldade de atendimento do princípio da conexão espacial, esse instrumento tem sido muito importante para a manutenção das UC e tem contribuído para compensar a perda de biodiversidade, não sendo o caso de extingui-lo e sim aprimorá-lo (Leite, 2015).

$\mathrm{Na}$ Espanha a compensação ambiental não monetária é prevista em relatórios de impacto ambiental que detalham os investimentos no meio ambiente assumidos pelos empreendedores que, em teoria, aproximam-se dos princípios da proporcionalidade e possuem uma conexão espacial e funcional com o hábitat perdido. Porém, conforme Villarroya \& Puig (2010), apenas 9\% desses relatórios descreveram as medidas de compensação a serem adotadas e, dentre os que especificaram, em geral as medidas são genéricas, mesmo em se tratando de projetos com diferentes graus de impacto e em diferentes regiões, demonstrando uma baixa efetividade do instrumento.

No Brasil, o EIA/RIMA também prevê a adoção de medidas compensatórias não monetárias e os resultados não têm sido diferentes dos encontrados por Villarroya \& Puig (2010). De acordo com o Ministério Público da União - MPU (2004), algumas falhas normalmente encontradas nos EIA/RIMA em relação a compensação ambiental foram:

- propostas iniciais de compensação de perdas baseadas em diagnósticos ambientais inadequados;

- ausência de informações detalhadas acerca dos recursos financeiros destinados aos programas e projetos ambientais;

- escassez de informações relacionadas às fontes dos recursos destinados à implantação dos compromissos de compensação ambiental.

Conforme Gastineau \& Taugourdeau (2014), nenhum dos métodos de compensação ambiental, ecológico e monetário é perfeito, destacando-se a dificuldade de ambos na determinação da equivalência entre os danos ambientais e a compensação adequada. Na compensação monetária, Zafonte \& Hampton (2007) sugerem que, sob certas condições, a valoração ambiental proporciona uma aproximação aceitável do valor a ser compensado. Outra palavra positiva sobre a compensação monetária foi dada por Flores \& Thacher (2002), destacando as potenciais ineficiências econômicas que poderiam ocorrer na distribuição dos custos e benefícios dos projetos compensatórios, caso a cobrança monetária fosse excluída.

\section{CONCLUSÕES}

A normativa legal que estabelece as diretrizes para a aplicação dos recursos da compensação ambiental é flexível e permite uma ampla mobilidade do órgão ambiental na tomada de decisão, seja na definição da UC beneficiada pelos recursos, seja no local de implantação de uma nova UC. Esses recursos têm sido aplicados nos mesmos biomas que sofrem os impactos dos empreendimentos licenciados, obedecendo ao princípio da conexão funcional. Porém os recursos são aplicados em municípios distintos do local do empreendimento, não sendo efetiva a aplicação do princípio da conexão espacial no uso dos recursos da compensação.

\section{STATUS DA SUBMISSÃO}

Recebido: 19 nov., 2015

Aceito: 7 set., 2016 
AUTOR(ES) PARA CORRESPONDÊNCIA

\section{Alexandre Nascimento de Almeida}

Departamento de Gestão Ambiental, Faculdade UnB de Planaltina - FUP, Universidade de Brasília - UnB, Área Universitária, 1, Vila Nossa Senhora de Fátima, CEP 73300-000, Planaltina, DF, Brasil

e-mail: alexalmeida@unb.br

\section{REFERENNCIAS}

Barbieri JC. Gestão ambiental empresarial. 2. ed. São Paulo: Saraiva; 2007.

Bechara E. Uma contribuição ao aprimoramento do Instituto da Compensação Ambiental previsto na Lei 9.985/2000. São Paulo; 2007.

Bezerra EGL. Biodiversity offsets in national (Brazil) and regional $(E U)$ manda-tory arrangements: towards an international regime? Washington, D.C.: BBOP; 2007 [citado em 2014 nov 5]. Disponível em: http://bbop. forest-trends.org/documents.

Brasil. Lei $n^{\circ} 6.938$, de 31 de agosto de 1981. Dispõe sobre a Política Nacional do Meio Ambiente, seus fins e mecanismos de formulação e aplicação, e dá outras providências. Diário Oficial da República Federativa do Brasil, Brasília, DF (1981 set.).

Brasil. Lei $n^{\circ}$ 9.985, de 18 de julho de 2000. Regulamenta o art. 225, $\$ 1^{\circ}$, incisos I, II, III e Vll da Constituição Federal, institui o Sistema Nacional de Unidades de Conservação da Natureza e dá outras providências. Diário Oficial da República Federativa do Brasil, Brasília, DF (2000 jul.).

Brasil. Resolução CONAMA no 371, de 5 de abril de 2006. Estabelece diretrizes aos órgãos ambientais para o cálculo, cobrança, aplicação, aprovação e controle de gastos de recursos advindos de compensação ambiental e dá outras providências. Diário Oficial da República Federativa do Brasil, Brasília, DF (2006 abr.).

Cole SG. Equity over efficiency: a problem of credibility in scaling resource-based compensatory? Journal of Environmental Economics and Policy 2013; 2(1): 93-117. http://dx.doi.org/10.1080/21606544.2013.764616.

Cowell R. Stretching the limits: environmental compensation, habitat creation and sustainable development. Transactions of the Institute of British 1997; 22(3): 297-306.

Darbi M, Ohlenburg H, Herberg A, Wende W, Skambracks $\mathrm{D}$, Herbert M. International approaches to compensation for impacts on biological diversity: final report. Dresden: Leibniz Institute of Ecological and Regional Development; 2009.

Domingues JM, Carneiro ASJ. A compensação ambiental prevista pelo Sistema Nacional de Unidades de Conservação
(SNUC): a ADI No 3.378 e o Decreto ${ }^{\circ}$ 6.848/09. Revista Direito FGV 2010; 493-502.

Dunford RW, Ginn TC, Desvousges WH. The use of habitat equivalency analysis in natural resource damage assessments. Ecological Economics 2004; 48(1): 49-70. http://dx.doi.org/10.1016/j.ecolecon.2003.07.011.

Faria ID. Compensação Ambiental: os fundamentos e as normas, a gestão e os conflitos. Brasília: Consultoria Legislativa do Senado Federal; 2008. Texto para discussão 43.

Flores NE, Thacher J. Money, who needs it? Natural resource damage assessment. Contemporary Economic Policy 2002; 20(2): 171-178. http://dx.doi.org/10.1093/ cep/20.2.171.

Gastineau P, Taugourdeau E. Compensating for environmental damages. Ecological Economics 2014; 97: 150-161. http:// dx.doi.org/10.1016/j.ecolecon.2013.11.008.

Gurgel H, Hargrave J, França F, Holmes RM, Ricarte FM, Dias BFS et al. Unidades de conservação e o falso dilema entre conservação e desenvolvimento. Boletim Regional. Urbano e Ambiental 2009; 3: 109-120.

Instituto Brasileiro de Geografia e Estatística - IBGE. Cidades. Rio de Janeiro; 2014 [citado em 2014 set 17]. Disponível em: http://www.ibge.gov.br.

Instituto Brasileiro do Meio Ambiente e dos Recursos Naturais Renováveis - IBAMA. Licenciamento ambiental. Brasília; 2014 [citado em 2014 ago 25]. Disponível em: http://www.ibama.gov.br.

Instituto Chico Mendes de Conservação da Biodiversidade - ICMBIO. Unidades de conservação. Brasília; 2014 [citado em 2014 ago 11]. Disponível em: http://www.icmbio.gov.br.

Leite FAS. Desafios e perspectivas da compensação ambiental para a criação e manutenção de unidades de conservação [dissertação]. Programa de Pós-graduação em Engenharia Ambiental, Universidade Federal de Ouro Preto, Ouro Preto, 2015.

Leoneti AB, Prado EL, Oliveira SVWB. Saneamento básico no Brasil: considerações sobre investimentos e sustentabilidade para o século XXI. Revista Administração Pública 2011; 45(2): 331-348. http://dx.doi.org/10.1590/ S0034-76122011000200003.

Martin-Ortega J, Brouwer R, Aiking H. Application of a value based equivalency method to assess environmental damage compensation under the European Environmental Liability Directive. Journal of Environmental Management 2011; 92(6): 1461-1470

Mckenney BA, Kiesecker JM. Policy development for biodiversity offsets: a review of offset frameworks. Environmental Management 2010; 45(1): 165-176. PMid:19924472. http://dx.doi.org/10.1007/s00267-0099396-3.

Medeiros R, Young CEF. Contribuição das unidades de conservação brasileiras para a economia nacional: relatório final. Brasília: UNEP-WCMC; 2011. 
Ministério Público da União - MPU. Deficiências em estudos de impacto ambiental: síntese de uma experiência. Brasília: Escola Superior do Ministério Público; 2004.

Pestana MH, Gageiro JN. Análise de dados para ciências sociais: a complementariedade do SPSS. Lisboa: Edições Silabo; 2005.

Quétier F, Regnery B, Levrel H. No net loss of biodiversity or paper offsets? A critical review of the French no net loss policy. Environmental Science \& Policy 2014; 38: 120-131. http://dx.doi.org/10.1016/j.envsci.2013.11.009.

Ribeiro MC, Metzger JP, Martensen AC, Ponzoni FJ, Hirota MM. The Brazilian Atlantic Forest: how much is left, and how is the remaining forest distributed: Implications for conservation. Biological Conservation 2009; 142(6): 11411153. http://dx.doi.org/10.1016/j.biocon.2009.02.021.

Rundcrantz K. Environmental compensation in Swedish road planning. European Environment 2006; 16(6): 350 367. http://dx.doi.org/10.1002/eet.429.

Sánchez LE. Avaliação de impacto ambiental: conceitos e métodos. São Paulo: Oficina de Textos; 2008.

Sociedade Brasileira para o Progresso da Ciência - SBPC, Academia Brasileira de Ciências - ABC. O código Florestal e a Ciência: contribuições para o diálogo. 2. ed. São Paulo: SBPC/ABC; 2012.

Suvantola L. Enviromental offset arrangements in biodiversity conservation: new instruments for the protection of biodiversity and biosafety Helsinki. Finland: Nordic Environmental Law Network Workshop; 2005.

Ten Kate K, Bishop J, Bayon R. Biodiversity offset: views experience and the bussines case. Gland: IUCN; 2004.

Ter Mors E, Terwel BW, Daamen DDL. The potential of host community compensation in facility siting. International
Journal of Greenhouse Gas Control 2012; 115: 130-138. http://dx.doi.org/10.1016/j.ijggc.2012.07.002.

The IUCN Red List of Threatened Species - IUCN. Independent report on biodiversity offsets. Cambridge: The Biodiversity Consultancy; 2012.

Villarroya A, Puig J. Ecological compensation and Environmental Impact Assessment in Spain. Environmental Impact Assessment Review 2010; 30(6): 357-362. http:// dx.doi.org/10.1016/j.eiar.2009.11.001.

World Database on Protected Areas - WDPA. 2009 [citado em 2014 nov 20]. Disponível em: http://www.wdpa.org/ Xie R, Pang Y, Li Z, Zhang N, Hu F. Eco-compensation in multi-district river networks in North Jiangsu, China. Environmental Management 2013; 51(4): 874-881. PMid:23229829. http://dx.doi.org/10.1007/s00267-0129992-5.

Zaal MP, Terwel BW, Ter Mors E, Daamen DDL. Monetary compensation can increase public support for the siting of hazardous facilities. Journal of Environmental Psychology 2014; 37: 21-30. http://dx.doi.org/10.1016/j. jenvp.2013.11.002.

Zafonte MC, Hampton S. Exploring welfare implications of resource equivalency analysis in natural resource damage assessments. Ecological Economics 2007; 61(1): 134-145. http://dx.doi.org/10.1016/j.ecolecon.2006.02.009. 